ARTICLE

Received 10 Aug 2012 | Accepted 28 Dec 2012 | Published 29 Jan $2013 \quad$ DOl: 10.1038/ncomms2426

\title{
The essential role of carefully optimized synthesis for elucidating intrinsic material properties of $(\mathrm{Ga}, \mathrm{Mn}) \mathrm{As}$
}

\author{
P. Němec ${ }^{1}$, V. Novák², N. Tesařová1, E. Rozkotová1, H. Reichlová1,2, D. Butkovičová', F. Trojánek¹, K. Olejník², \\ P. Malý1, R.P. Campion ${ }^{3}$, B.L. Gallagher ${ }^{3}$, Jairo Sinova ${ }^{2,4} \&$ T. Jungwirth ${ }^{2,3}$
}

$(\mathrm{Ga}, \mathrm{Mn}) \mathrm{As}$ is at the forefront of spintronics research exploring the synergy of ferromagnetism with the physics and the technology of semiconductors. However, the electronic structure of this model spintronics material has been debated and the systematic and reproducible control of the basic micromagnetic parameters and semiconducting doping trends has not been established. Here we show that seemingly small departures from the individually optimized synthesis protocols yield non-systematic doping trends, extrinsic charge and moment compensation, and inhomogeneities that conceal intrinsic properties of ( $\mathrm{Ga}, \mathrm{Mn})$ As. On the other hand, we demonstrate reproducible, well controlled and microscopically understood semiconducting doping trends and micromagnetic parameters in our series of carefully optimized epilayers. Hand-in-hand with the optimization of the material synthesis, we have developed experimental capabilities based on the magneto-optical pumpand-probe method that allowed us to simultaneously determine the magnetic anisotropy, Gilbert damping and spin stiffness constants from one consistent set of measured data.

\footnotetext{
${ }^{1}$ Faculty of Mathematics and Physics, Charles University in Prague, Ke Karlovu 3, 12116 Prague 2, Czech Republic. ${ }^{2}$ Institute of Physics ASCR, v.v.i., Cukrovarnická 10, 16253 Praha 6, Czech Republic. ${ }^{3}$ School of Physics and Astronomy, University of Nottingham, Nottingham NG7 2RD, United Kingdom. ${ }^{4}$ Department of Physics, Texas A\&M University, College Station, Texas 77843-4242, USA. Correspondence and requests for materials should be addressed to T.J. (email: jungw@fzu.cz).
} 
U nder equilibrium growth conditions, the incorporation of magnetic $\mathrm{Mn}$ ions into GaAs is limited to $\sim 0.1 \%$. To circumvent the solubility problem, a non-equilibrium, low-temperature molecular-beam-epitaxy (LT-MBE) technique was employed, which led to first successful growths of (Ga,Mn)As ternary alloys with more than $1 \% \mathrm{Mn}$ and to the discovery of ferromagnetism in these materials ${ }^{1-4}$. The compounds qualify as ferromagnetic semiconductors to the extent that their magnetic and other properties can be altered by the usual semiconductor electronics engineering variables, such as doping, electric fields, or light. (Ga,Mn)As has become a test-bed material for spintronic research, in particular for exploring known and discovering new magneto-transport and magneto-optical (MO) phenomena. (For more details on $(\mathrm{Ga}, \mathrm{Mn}) \mathrm{As}$ spintronics research, see Supplementary Note 1).

In ferromagnetic (Ga,Mn)As, the theory of spin-orbit-coupled GaAs bands modified by the exchange splitting and alloying effects of randomly distributed $\mathrm{Mn}_{\mathrm{Ga}}$ acceptors/local-moments represents a common basis for describing its semiconducting and ferromagnetic properties ${ }^{5}$. The kinetic-exchange model implementation of this valence band theory and the microscopic tight-binding Anderson approach or abinitio density functional theory can all be shown to be mutually consistent on the level of atomic and orbital resolved band structure $^{6}$. (For more details on the theory of (Ga,Mn)As, see Supplementary Figs S1-S3, and Supplementary Note 2).

In a recurring alternative proposal, the Fermi level in ferromagnetic materials with $\sim 10^{20}-10^{21} \mathrm{~cm}^{-3}$ Mn-acceptor densities is assumed to still reside in a narrow impurity band detached from the valence band, that is, the band structure keeps the form closely reminiscent of a single isolated $\mathrm{Mn}_{\mathrm{Ga}}$ impurity level ${ }^{7-9}$. Several phenomenological variants of the impurity band model have been proposed whose assumed spectroscopic characteristics are, however, mutually inconsistent and have not been recreated from microscopic band structure theories ${ }^{6}$. Since to date no framework has been introduced that would allow to test the validity of the impurity band model by comparing microscopic atomic orbital based theory calculations to experiment, it is primarily the experimental findings which have served as a basis for postulating the impurity band picture. A common feature of these experimental reports is the limited attention paid to the details of the synthesis of this highly nonequilibrium alloy 7,9 . As we show in this paper, careful material optimization is, however, of central importance. Small departures from optimized sample synthesis obscure the doping trends and experimental determination of intrinsic micromagnetic parameters of the material. The justification of the impurity band picture by the experiments in unoptimized materials is therefore unreliable. This leaves the impurity band models without an adequate microscopic theory or experimental support. On the other hand, the valence band model is linked in a transparent way to density functional or tight-binding Anderson microscopic theories, as pointed out above. Simultaneously, the microscopically justified theory provides consistent predictions for the broad range of semiconducting doping trends and micromagnetic parameters, we report below on the entire series of the optimized $(\mathrm{Ga}, \mathrm{Mn})$ As materials. Our work, therefore, leads to the conclusion that there is no physics grounds for the 'battle of the bands ${ }^{10}$ in (Ga,Mn)As.

\section{Results}

Optimized synthesis of (Ga,Mn)As epilayers. The essential importance of the optimization of the $(\mathrm{Ga}, \mathrm{Mn}) \mathrm{As}$ synthesis is illustrated in Fig. 1, where we plot, side-by-side, basic electrical and magnetic characteristics of two medium, $7 \%$ nominally
Mn-doped epilayers. (The determination of the nominal Mn concentration in the as-grown epilayers is detailed in Methods). The left column figures (Fig. 1a-c) show data measured on a material, which we prepared under carefully optimized conditions for the given nominal Mn doping. The sample has sharp Curie point singularities in magnetization and electrical transport typical of uniform, high-quality itinerant ferromagnets (Fig. 1a $)^{11}$. Magnetization precession damping factor and spinwave resonances (SWRs) obtained from MO measurements (Fig. 1b,c) confirm the high magnetic quality of the material. As we discuss in detail below, the initial decrease of the damping factor with frequency followed by a frequency-independent part (Fig. 1b) is typical of uniform ferromagnets and allows us to accurately infer the intrinsic Gilbert damping constant $^{12}$. Similarly, the observed Kittel SWR modes of a uniform ferromagnet (Fig. 1c) allow us to measure accurately the magnetic anisotropy and spin stiffness parameters of (Ga,Mn)As, as detailed below.

The right column data (Fig. 1d-f) were measured on a $7 \% \mathrm{Mn}$ doped epilayer differing from the sample of the left column in
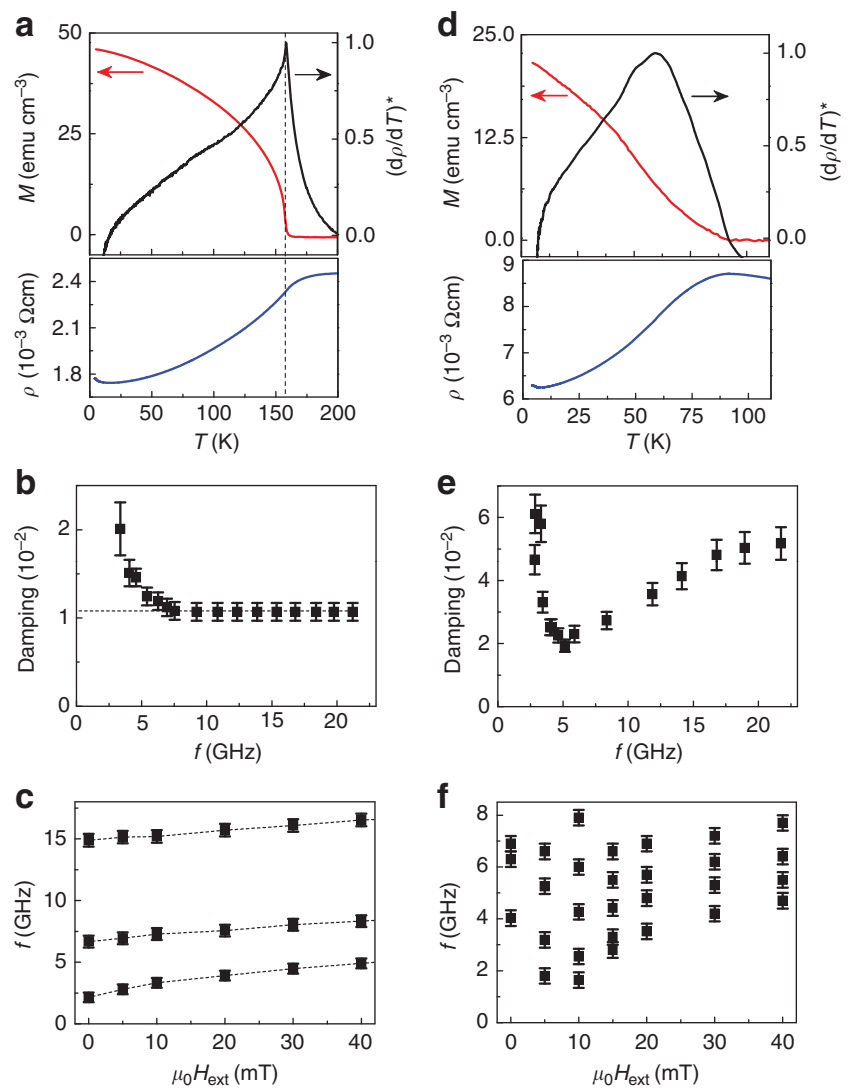

Figure 1 | Demonstration of the essential role of the careful optimization of (Ga,Mn)As synthesis. (a), Magnetization $M$ (red line), temperature derivative of the resistivity normalized to the peak value $(\mathrm{d} \rho / \mathrm{d} T)^{\star}$ (black line) and resistivity $\rho(T)$ (blue line) of an optimized 20-nm-thick epilayer with 7\% nominal Mn doping. (b,c) Frequency dependence of the damping factor and field dependence of the spin-wave resonant frequencies of the same sample. Dotted line in $\mathbf{b}$ highlights the Gilbert constant value; dotted lines in panel $\mathbf{c}$ highlight the $n=0,1$ and 2 Kittel spin-wave modes. (d-f) Same as panels a-c for a material differing by having only one of the synthesis parameters not optimized (epilayer thickness of $500 \mathrm{~nm}$ too large). Gilbert constant and Kittel modes cannot be inferred from e and $\mathbf{f}$. The error bars correspond to s.d. and depict the overall uncertainty in the determination of the displayed value from the experimentally measured data. 
only one of the synthesis parameters not being optimized. As we discuss below, the stoichiometry, substrate growth temperature, post-growth annealing temperature and time and epilayer thickness are among the key synthesis parameters. (For more details on our materials synthesis, see Methods.) All these parameters were equally carefully optimized in the two samples, except for the epilayer thickness. In the medium and high Mndoped samples, full material optimization is possible only for film thicknesses $\lesssim 50 \mathrm{~nm}$. The epilayer whose measurements are shown in the right panels of Fig. 1 is $500 \mathrm{~nm}$ thick. Its magnetization and transport Curie point singularities are largely smeared out, the damping factor is strongly frequency dependent ${ }^{13}$, and alternating number of SWRs is observed with increasing applied field whose spacings are inconsistent with Kittel modes. Clearly, the material is non-uniform, the magnetization and transport data indicate strong moment and charge compensation by extrinsic impurities, and for this material it is impossible to reliably extract any of the intrinsic micromagnetic parameters of $(\mathrm{Ga}, \mathrm{Mn}) \mathrm{As}$.

In Fig. 2, we illustrate that even in films thinner than $50 \mathrm{~nm}$, apparently small changes in the remaining key synthesis parameters can dramatically affect the material quality. It is
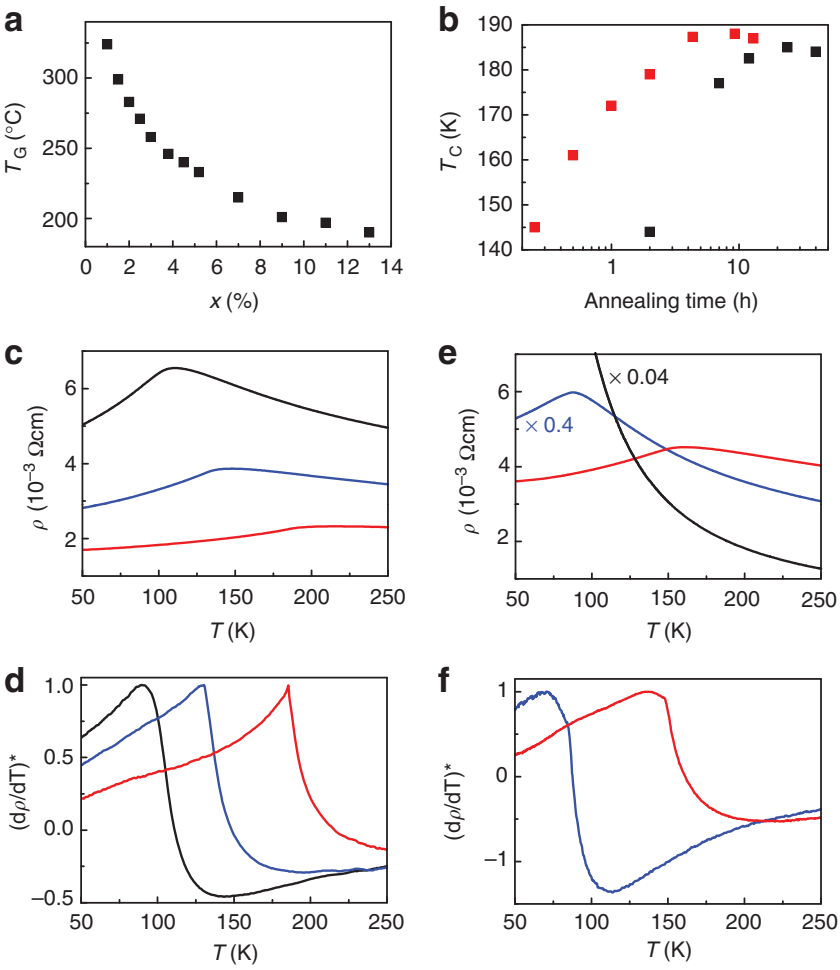

Figure 2 | Individual optimization for each nominal Mn doping of the growth temperature and of the annealing temperature and time.

(a) Optimal growth temperature $T_{\mathrm{G}}$ as a function of the nominal Mn-doping $x$. (b) Dependence of the Curie temperature $T_{C}$ on the annealing time for two annealing temperatures $T_{\mathrm{A}}=160^{\circ} \mathrm{C}$ (red points) and $140^{\circ} \mathrm{C}$ (black points) in a $15-\mathrm{nm}$-thick $(\mathrm{Ga}, \mathrm{Mn})$ As epilayer with $13 \%$ nominal $\mathrm{Mn}$ doping grown at optimal $T_{G}$. (c,d) Resistivity $\rho(T)$ and temperature derivative of the resistivity normalized to the peak value $(\mathrm{d} \rho / \mathrm{d} T)^{\star}$ in the $x=13 \%$ epilayer grown at optimal $T_{\mathrm{G}}$ in the as-grown state (black line), for optimal $T_{\mathrm{A}}$ and annealing time $0.5 \mathrm{~h}$ (blue line) and for optimal $T_{\mathrm{A}}$ and optimal annealing time of $8 \mathrm{~h}$ (red line). (e,f) Same as panels $\mathbf{c}$ and $\mathbf{d}$ for a $x=13 \%$ epilayer grown at $10{ }^{\circ} \mathrm{C}$ below optimal $T_{\mathrm{G}} ; \rho(T)$ for the as-grown state and for the annealing time $0.5 \mathrm{~h}$ are multiplied by the numerical factors depicted in the figure for clarity; $(\mathrm{d} \rho / \mathrm{d} T)^{\star}$ is not plotted for the as-grown insulating and paramagnetic sample. broadly accepted that staying near the 1:1 stoichiometric As: $(\mathrm{Ga}+\mathrm{Mn})$ ratio is favourable for the LT-MBE growth of $(\mathrm{Ga}, \mathrm{Mn}) \mathrm{As}^{14,15}$. In Fig. 2a, we plot our optimal growth temperature $T_{\mathrm{G}}$ for the stoichiometric growth as a function of the nominal Mn-doping $x$. (We used the GaAs band-edge spectrometer to measure the substrate temperature and the predictive substrate heater control to stabilize the $T_{\mathrm{G}}$ during the growth). Note that the optimal $T_{\mathrm{G}}$ remains near (from the lower temperature side) the $2 \mathrm{D} / 3 \mathrm{D}$ growth-mode boundary, which implies its strong dependence on $x$. In Fig. 2b, we plot the Curie temperature $T_{\mathrm{C}}$ as a function of the annealing time for the optimal $T_{\mathrm{G}}=190{ }^{\circ} \mathrm{C}$ for the $13 \%$ Mn-doped sample and for two annealing temperatures. One is the optimal annealing temperature $T_{\mathrm{A}}=160^{\circ} \mathrm{C}$ and the other one is $20^{\circ} \mathrm{C}$ lower. Clearly, the record $T_{\mathrm{C}}=188 \mathrm{~K}$ sample is obtained by optimizing simultaneously the annealing time and $T_{\mathrm{A}}$. Figure $2 c$,d illustrate how the increasing $T_{\mathrm{C}}$ is accompanied by the improving material quality (reduction of extrinsic compensation and sample inhomogeneity) over the annealing time for optimal $T_{\mathrm{G}}$ and $T_{\mathrm{A}}$. To highlight the crucial importance of the optimal $T_{\mathrm{G}}$ during the growth, we show in Fig. 2e,f the same annealing sequence measurements as in Fig. $2 c$,d on a $13 \%$-doped sample grown at a temperature of only $10^{\circ} \mathrm{C}$ below the optimal $T_{\mathrm{G}}$. In striking contrast to the material grown at the optimal $T_{\mathrm{G}}$, the sample is insulating and paramagnetic in the as-grown state. Ferromagnetism and metallic conduction can be recovered by annealing, however, the compensation and inhomogeneity cannot be removed and the ferromagnetic transition temperature remains $10{ }^{\circ} \mathrm{C}$ below the $T_{\mathrm{C}}$ of the sample grown at the optimal $T_{\mathrm{G}}$. Similarly lower-quality samples are obtained by growing at higher than the optimal $T_{\mathrm{G}}$.

Figures 1 and 2 illustrate the following general conclusions we can draw from our extensive material optimization studies. Inferring doping trends in basic material properties of $(\mathrm{Ga}, \mathrm{Mn}) \mathrm{As}$ from sample series mixing as-grown and annealed materials, as has been often the case in the literature ${ }^{7,9}$, is unsuitable as the quality of the samples may vastly vary in such a series. Chosing one a priori-fixed $T_{\mathrm{G}}, T_{\mathrm{A}}$ and annealing time for a range of $\mathrm{Mn}$ dopings, as is also common in the literature ${ }^{7,9,16,17}$, is unlikely to produce a high-quality, uniform and uncompensated $(\mathrm{Ga}, \mathrm{Mn}) \mathrm{As}$ material even for one of the considered dopings and is bound to produce lower quality samples for most of the studied $\mathrm{Mn}$ dopings. Finally, reliable measurements of intrinsic semiconducting and magnetic properties on optimized $(\mathrm{Ga}, \mathrm{Mn})$ As samples require exceedingly long annealing times for film thicknesses $\gtrsim 50 \mathrm{~nm}$ and are unachievable in $\sim 100 \mathrm{~nm}$ and thicker films by the known approaches to the $(\mathrm{Ga}, \mathrm{Mn}) \mathrm{As}$ epilayer synthesis.

Doping trends in the series of optimized (Ga,Mn)As epilayers. After finding the optimal growth and post-growth conditions for each individual nominal doping, we obtained a series of comparably high-quality samples spanning a wide range of $\mathrm{Mn}$ dopings. At nominal dopings below $\sim 0.1 \%$, the materials from the series are paramagnetic, strongly insulating, showing signatures of the activated transport corresponding to valence band -impurity band transitions at intermediate temperatures, and valence band-conduction band transitions at high temperatures (Fig. 3a). For higher nominal dopings, $0.5 \gtrsim x \gtrsim 1.5 \%$, no clear signatures of activation from the valence band to the impurity band are seen in the transport, confirming that the bands start to overlap and mix, yet the materials remain insulating. At $x \approx 1.5 \%$, the low-temperature conductivity of the film increases abruptly by several orders of magnitude (Fig. 3b), and the system turns into a degenerate semiconductor. The onset of ferromagnetism 
occurs already on the insulating side of the insulator-to-metal transition at $x \approx 1 \%$. All metallic samples within the series have sharp Curie point singularities (Fig. 3c).

The hole density $p$ measured from the high-field Hall effect (see Supplementary Fig. S4, Supplementary Equation S1, and Supplementary Note 4 for more details) increases with increasing doping in the optimized materials (Fig. 3d). Similarly, the magnetization and $T_{\mathrm{C}}$ steadily increase with $x$ (Fig. 3e,f). Assuming $4.5 \mu_{\mathrm{B}}$ per $\mathrm{Mn}$ atom ${ }^{18}$ ( $\mu_{\mathrm{B}}$ is the Bohr magneton), we can estimate the density $N_{\mathrm{Mn}}$ of uncompesated $\mathrm{Mn}_{\mathrm{Ga}}$ moments from the magnetization data (see left $y$ axis in Fig. 3e). As there is no apparent deficit of $p$ compared with $N_{\mathrm{Mn}}$ and as the interstitial Mn impurity, which is the main extrinsic defect, compensates one local moment but two holes, we conclude that interstitial Mn is completely (within the experimental error) removed in our optimally synthesized epilayers. Hence, our series of (Ga,Mn)As materials have well-controlled characteristics, showing a systematic trend of increasing magnetization with increasing $x$, increasing $T_{\mathrm{C}}$ (reaching $188 \mathrm{~K}$ ) and increasing hole density. The materials have no measurable charge or moment compensation of the substitutional $\mathrm{Mn}_{\mathrm{Ga}}$ impurities and have a large degree of uniformity reflected by sharp Curie point singularities. We point out that this phenomenology is in striking contrast to the nonsystematic doping trends in semiconducting and magnetic properties reported, for example, in Dobrowolska et al. ${ }^{9}$ on the mixed series of as-grown and annealed, 100-nm-thick
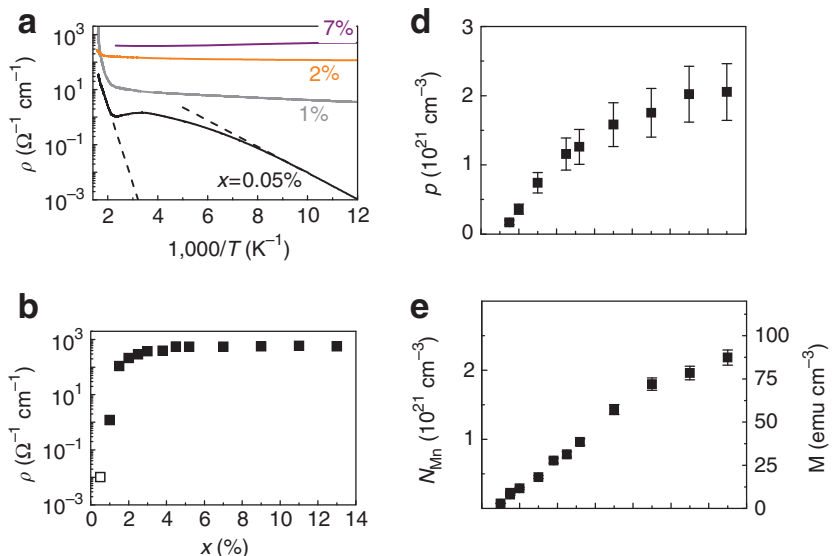

C
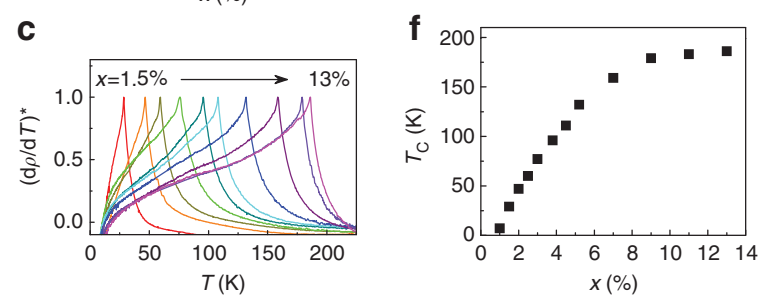

Figure 3 | Doping trends in the series of optimized ( $\mathrm{Ga}, \mathrm{Mn})$ As epilayers. (a) Temperature dependence of the conductivity $\sigma(T)$ of optimized $(\mathrm{Ga}, \mathrm{Mn})$ As epilayers with depicted nominal $\mathrm{Mn}$ doping. Dashed lines indicate the activated parts of $\sigma(T)$ of the insulating paramagnetic ( $\mathrm{Ga}, \mathrm{Mn}$ ) As with $0.05 \% \mathrm{Mn}$ doping, corresponding to the $\mathrm{Mn}$ acceptor level and the band gap, respectively. (b) Conductivity at $4 \mathrm{~K}$ as a function of the nominal $\mathrm{Mn}$ doping. Open symbol corresponds to a paramagnetic sample. (c) Sharp Curie point singularities in the temperature derivative of the resistivity in the series of optimized ferromagnetic $(\mathrm{Ga}, \mathrm{Mn}) \mathrm{As}$ epilayers with metallic conduction and $x=0.5,2,2.5,3,3.8,4.5,5.2,7,9$ and $13 \%$. (d-f) Hole density $p$, magnetization $M$ and corresponding $M n$ moment density $N_{\mathrm{Mn}}$ and Curie temperature $T_{\mathrm{C}}$ as a function of the nominal $\mathrm{Mn}$ doping in the series of optimized $(\mathrm{Ga}, \mathrm{Mn})$ As epilayers.
(Ga,Mn)As epilayers prepared without individually optimizing $T_{\mathrm{G}}, T_{\mathrm{A}}$ and the annealing time.

Micromagnetic parameters of (Ga,Mn)As. Figure 4 demonstrates that the intrinsic micromagnetic parameters of (Ga,Mn)As measured on our carefully optimized materials show also a smooth monotonous doping dependence. As detailed below, their values are characteristic of common band ferromagnets and all the semiconducting and magnetic properties summarized in Figs 3 and 4 are consistent with the microscopically established electronic structure of (Ga,Mn)As (refs 5,6). We point out that we have confirmed the control and reproducibility of the above material properties of $(\mathrm{Ga}, \mathrm{Mn})$ As on the individually optimized films by multiple material synthesis experiments at a given $\mathrm{Mn}$ doping in two distinct MBE laboratories in Prague and in Nottingham. This is a positive observation, highly non-trivial for the heavily doped non-equilibrium grown semiconductor, which is essential for the reliable understanding and utilizing the material. Not surprisingly for the highly non-equilibrium synthesis, the growth parameters quoted in this paper should not be taken as a universal recipe for $(\mathrm{Ga}, \mathrm{Mn})$ As. The optimal growth and post-growth annealing conditions may vary from one to the other experimental system to an extent, which for our two MBE laboratories is not large but may be beyond the straightforward cross-calibration error bars.

A more than an order of magnitude experimental scatter and a lack of any clear trend as a function of Mn doping can be found in the previous literature for the Gilbert damping and spin stiffness constants (Supplementary Note 3$)^{19-28}$. Significant variations can be also found in the experimental magnetic anisotropy constants of (Ga,Mn)As (ref. 29). The disarray of the previously measured micromagnetic parameters reflects partly the lack of careful materials optimization but also the limitations of common magnetic characterization techniques when applied to the thin-film dilute-moment (Ga,Mn)As samples. In the remaining part of the paper, we therefore describe in more detail our experimental determination of the micromagnetic parameters of $(\mathrm{Ga}, \mathrm{Mn})$ As. Hand-in-hand with the optimization of the material synthesis, we have developed experimental capabilities based on the MO pump-and-probe method that allows us to simultaneously determine the magnetic anisotropy, Gilbert damping and spin stiffness constants from one consistent set of measured data. The possibility to excite and detect precession of ferromagnetic Mn moments in (Ga,Mn)As by this method has been extensively discussed in previous MO studies (see Methods and Supplementary Notes 6-9 for the detailed
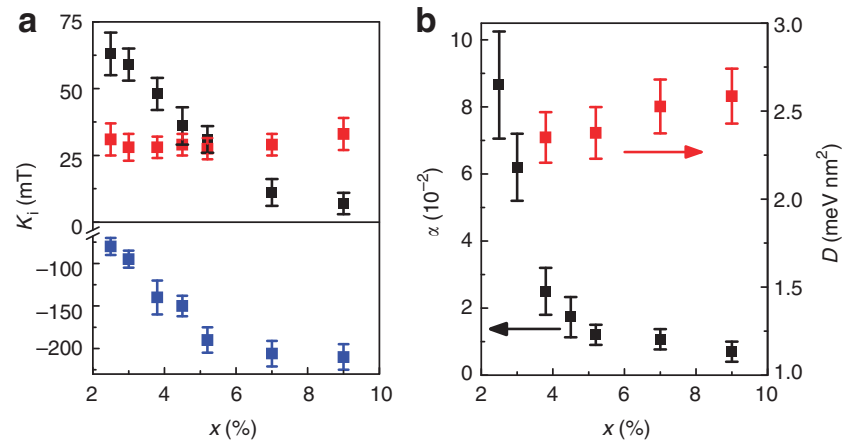

Figure 4 | Micromagnetic parameters of optimized epilayers of ferromagnetic $(\mathbf{G a}, \mathbf{M n})$ As. (a) Dependence of anisotropy fields $K_{\mathrm{c}}$ (black points), $K_{\mathrm{u}}$ (red points) and $K_{\text {out }}$ (blue points) on nominal $M n$ doping. (b) Dependence of the Gilbert damping constant $\alpha$ and the spin stiffness constant $D$ on nominal $M n$ doping. 
description of the method) ${ }^{30-41}$. All experiments presented below were performed at $15 \mathrm{~K}$.

The anisotropy constants, shown in Fig. 4a, were obtained combining three complementary measurements. In the first experiment, we measured the external magnetic field $H_{\text {ext }}$ dependence of the precession frequency $f$ of the time-resolved MO signal. In the studied (Ga,Mn)As/GaAs epilayers, the internal magnetic anisotropy fields are dominated by three components. The out-of-plane component $K_{\text {out }}$ is a sum of the thin-film shape anisotropy and the magnetocrystalline anisotropy due to the compressive growth strain in $(\mathrm{Ga}, \mathrm{Mn}) \mathrm{As}$. The cubic magnetocrystalline anisotropy $K_{\mathrm{C}}$ reflects the zinc-blende crystal structure of the host semiconductor. The additional uniaxial anisotropy component along the in-plane diagonal $K_{\mathrm{u}}$ is not associated with any measurable macroscopic strain in the epilayer. The precession frequency is given by,

$$
\begin{aligned}
f=\frac{g \mu_{\mathrm{B}}}{h} & \sqrt{\left(H_{\mathrm{ext}} \cos \left(\varphi-\varphi_{\mathrm{H}}\right)-2 K_{\mathrm{out}}+K_{\mathrm{c}}(3+\cos 4 \varphi) / 2+2 K_{\mathrm{u}} \sin ^{2}(\varphi-\pi / 4)+\Delta H_{n}\right)} \\
& \times \sqrt{\left(H_{\mathrm{ext}} \cos \left(\varphi-\varphi_{\mathrm{H}}\right)+2 K_{\mathrm{c}} \cos 4 \varphi-2 K_{\mathrm{u}} \sin 2 \varphi+\Delta H_{n}\right)},
\end{aligned}
$$

where $g$ is the Landé $g$-factor of Mn moments, $\varphi$ and $\varphi_{\mathrm{H}}$ are the in-plane magnetization and external magnetic field angles measured from the [100] crystal axis, and $\Delta H_{n}$ is the shift of the resonant field for the higher-index $n$ spin-wave modes with respect to the $n=0$ uniform precession mode. To uniquely determine the anisotropy constants, the field-dependent precession frequency measurements were complemented by MO experiments with variable polarization angle of the probe beam. The latter measurements allow us to precisely determine the angle of the equilibrium easy axis of the magnetization (Supplementary Note 7$)^{40,41}$. Finally, we confirmed the consistency of the obtained anisotropy constants by performing static measurements of magnetization hysteresis loops by the superconducting quantum interference device. The sign of $K_{\text {out }}$ implies that all studied $(\mathrm{Ga}, \mathrm{Mn}) \mathrm{As} / \mathrm{GaAs}$ materials are in-plane ferromagnets. Note that in the low-doped and high-doped samples with very stable in-plane easy-axes aligned with one of the main crystal directions, the dynamical MO experiments become unfeasible. The overall magnitudes of the anisotropy constants, as well as of the magnetization and $T_{\mathrm{C}}$, are consistently described by the microscopically established band theories of (Ga,Mn)As (ref. 5).

The magnetization precession damping factor, shown in Fig. $4 \mathrm{~b}$, is obtained by fitting the measured dynamical MO signal to Landau-Lifshitz-Gilbert (LLG) equations using the experimentally obtained magnetic anisotropy constants. The high accuracy of the LLG fits is demonstrated in Fig. 5a,b on data measured on the 5.2\%-doped sample. The obtained dependence of the damping factor on the external magnetic field applied along the [010] and [110] directions is shown in Fig. 5c. At smaller fields, the damping factor is not constant and shows a strong anisotropy with respect to the field angle. When plotted as a function of frequency, however, the dependence on the field-angle disappears, as shown in Fig. 5d. We can therefore conclude that the apparent anisotropy of the damping factor can in our materials be ascribed fully to the field-angle dependence via the precession frequency. In all our studied materials, the frequencyindependent Gilbert damping constant $\alpha$ is isotropic and can be accurately determined from MO data with precession frequencies $f \gtrsim 15 \mathrm{GHz}$. The MO damping factor phenomenology observed in Fig. 5 (and Fig. 1b) is consistent with corresponding MO measurements in uniform metal ferromagnets ${ }^{12}$.

We point out that in ferromagnetic resonance (FMR) experiments, the measurement frequency was limited typically to two values, $f=9$ and $35 \mathrm{GHz}$, which even in the optimized (Ga,Mn)As materials is not sufficient to reliably separate the
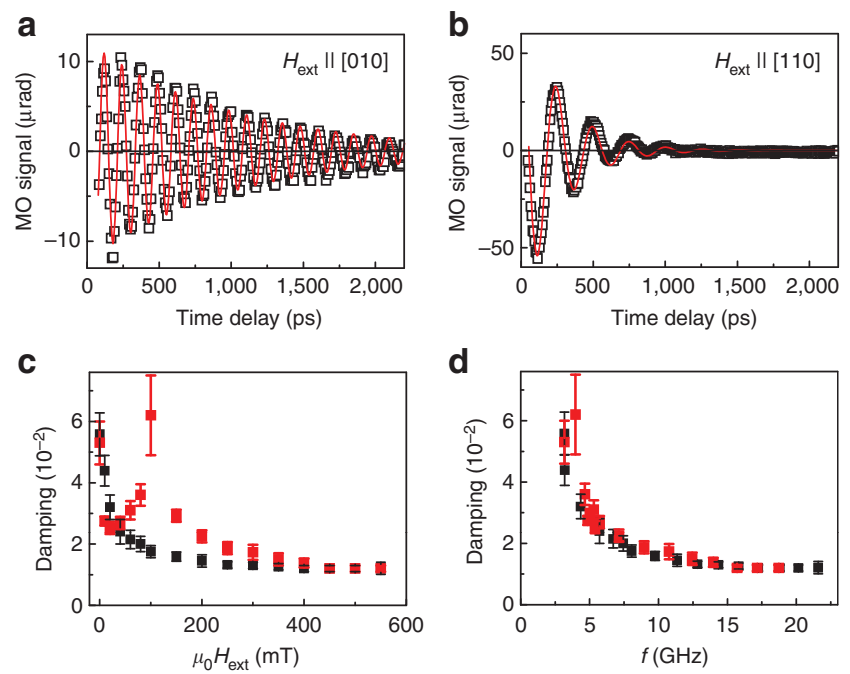

Figure 5 | Determination of the Gilbert damping constant. $(\mathbf{a}, \mathbf{b})$

Oscillatory part of the MO signal (points) measured in a 18-nm thick epilayer with 5.2\% nominal Mn doping for external magnetic field $\mu_{0} H_{\text {ext }}=100 \mathrm{mT}$ applied along the crystallographic directions [010] and [110]; lines are fits by the LLG equation. (c,d) Dependence of the magnetization precession damping factor on the external magnetic field and on the precession frequency for fields applied along the [010] (black points) and [110] (red points) crystallographic directions.

intrinsic Gilbert damping constant from the inhomogeneous broadening of the FMR line-width. The dynamical MO measurements, on the other hand, span a large-enough range of frequencies and allow us to extract a consistent set of frequencyindependent values of $\alpha$ for our series of optimized ferromagnetic $(\mathrm{Ga}, \mathrm{Mn})$ As materials. We find a systematic doping trend across the series in which the Gilbert constant decreases from $\sim 0.1$ to 0.01 when the nominal Mn doping increases from $\sim 2$ to $5 \%$, and then remains nearly constant (Fig. 4b). The magnitudes of $\alpha$ and the doping dependence are consistent with Gilbert damping constants in conventional transition metal ferromagnets. In metals, $\alpha$ typically increases with increasing resistivity and is enhanced in alloys with enhanced spin-orbit coupling ${ }^{42-44}$. Similarly, in our measurements in $(\mathrm{Ga}, \mathrm{Mn}) \mathrm{As}$, the increase of $\alpha$ correlates with a sizable increase of the resistivity in the lower Mn-doped samples. Also, the spin-orbit coupling effects tend to be stronger in the lower doped samples with lower filling of the valence bands and with the carriers closer to the metal-insulator transition $^{5}$. Theory ascribing magnetization relaxation to the kinetic-exchange coupling of $\mathrm{Mn}$ moments with holes residing in the disordered, exchange-split and spin-orbit-coupled valence band of ( $\mathrm{Ga}, \mathrm{Mn})$ As yields a comparable range of values of $\alpha$ as observed in our measurements ${ }^{19}$.

The MO pump-and-probe technique utilized in our work allows us in principle also for the direct measurement of the spin stiffness. However, one has to find the rather delicate balance between thin enough epilayers whose material quality can be optimized and thick enough films allowing the observation of the higher-index Kittel spin-wave modes ${ }^{45}$ of a uniform ferromagnet. For these modes, the spin stiffness parameter $D$ is directly obtained from the measured resonant fields,

$$
\Delta H_{n} \equiv H_{0}-H_{n}=D \frac{n^{2}}{L^{2}} \frac{\pi^{2}}{g \mu_{\mathrm{B}}},
$$

where $L$ is the thickness of the ferromagnetic film. The MO pump-and-probe technique has the key advantage here that, 
unlike FMR, it is not limited to odd index spin-wave modes ${ }^{45}$. The ability to excite and detect the $n=0,1$ and 2 resonances is essential for the observation of the Kittel modes in our optimized (Ga,Mn)As epilayers whose thickness is limited to $\sim 50 \mathrm{~nm}$.

In Fig. 6a, we show an example of the time-dependent MO signal measured in a 48 -nm-thick optimized epilayer with $7 \%$ nominal Mn doping. Three SWRs are identified in the sample with frequencies $f_{0}, f_{1}$ and $f_{2}$, as shown in Fig. 6b,c. The association of these SWRs with the Kittel modes, described by equation (2), is based on experiments shown in Fig. 6b-e. In Fig. $6 c$, we plot the dependence of the three detected precession frequencies on the external magnetic field applied along the [010] and [110] crystal axes. At saturation fields, which for the $7 \% \mathrm{Mn}$ doped sample are $\gtrsim 70 \mathrm{mT}$, the equilibrium magnetization vector is aligned with $H_{\text {ext }}$ and equation (1) with $\varphi=\varphi_{\mathrm{H}}$ can be used to fit the data. We emphasize that all six displayed dependences $f_{n}\left(H_{\text {ext }}\right)$ for $n=0,1$ and 2 , and $\varphi_{\mathrm{H}}=45^{\circ}$ and $90^{\circ}$ can be accurately fitted by one set of magnetic anisotropy constants. We can therefore use equation (2) to convert the measured frequency spacing of individual SWRs to $\Delta H_{n}$. In Fig. 6d, we show that $\Delta H_{n}$ in our optimized epilayers is proportional to $n^{2}$, as expected for the Kittel modes in homogeneous films.
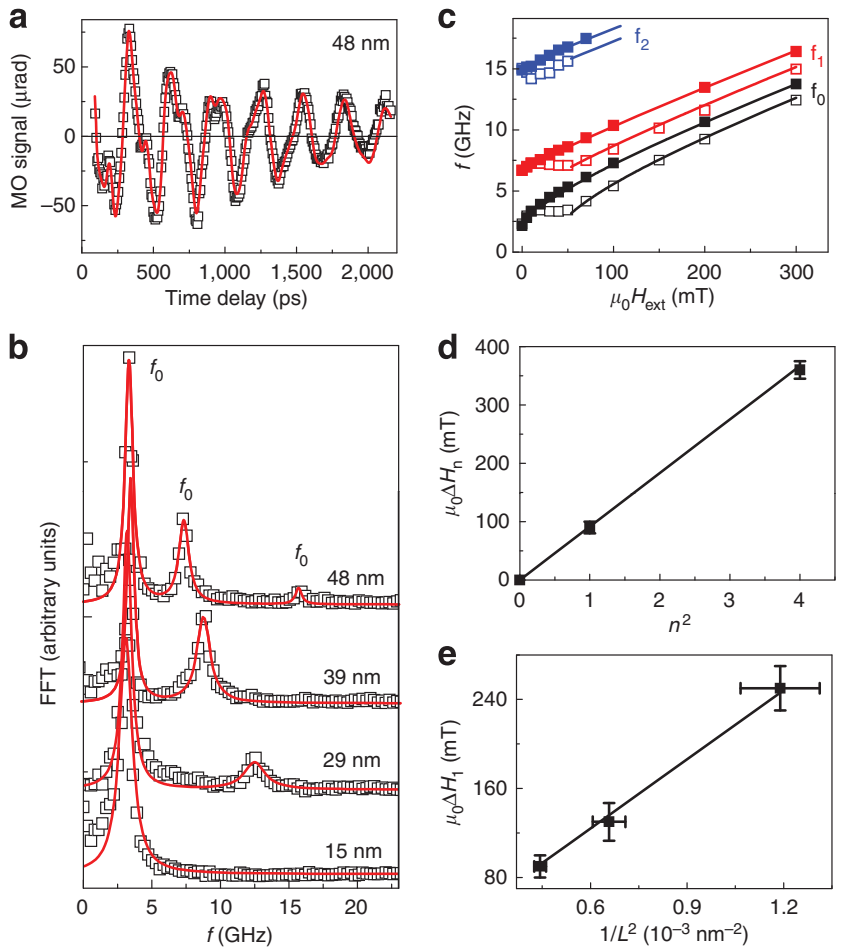

Figure 6 | Determination of the spin stiffness constant. (a) Oscillatory part of the MO signal (points) measured in a $48-n m$-thick epilayer with $7 \%$ nominal $\mathrm{Mn}$ doping for external magnetic field $\mu_{0} \mathrm{H}_{\text {ext }}=20 \mathrm{mT}$ applied along the [010] crystallographic direction; line is a fit by a sum of three damped harmonic functions. (b) Fourier spectra of oscillatory MO signals (points) measured for $\mu_{0} H_{\text {ext }}=20 \mathrm{mT}$ applied along the [010]

crystallographic direction in samples prepared by etching from the 48-nmthick epilayer. The curves are labelled by the film thicknesses, normalized and vertically off-set for clarity; lines are fits by a sum of Lorentzian peaks. (c) Dependence of the measured precession frequency on the magnetic field for fields along the crystallographic directions [010] (solid points) and [110] (open points) in the 48-nm-thick epilayer; lines are fits by equation (1). (d) Dependence of the measured mode spacing on square of the mode number in the 48-nm-thick epilayer. (e) Dependence of the spacing between the two lowest modes $\left(\Delta H_{1}\right)$ on the film thickness. Lines in $\mathbf{d}$ and $\mathbf{e}$ are fits by equation (2) with spin stiffness $D=2.43 \mathrm{meVnm}$.
The magnetic homogeneity and the applicability of equation (2) in our epilayers is further confirmed by the following experiments: We prepared three samples by etching the original 48-nm-thick (Ga,Mn)As film down to the thicknesses of 39,29 and $15 \mathrm{~nm}$, respectively. As seen in Fig. 6b, the frequency $f_{0}$ is independent of the film thickness, which confirms that it corresponds to the uniform precession mode and that the film is homogeneous, that is, the magnetic anisotropy constants do not vary across the width of the (Ga,Mn)As epilayer. Note that this is again in striking contrast with the vertical gradient of magnetic anisotropies reported, for example, in Son et $a l^{46}$ on a $\sim 500$-nm-thick unoptimized material. (For another direct evidence of the crucial role of the growth parameters on the material uniformity, compare also results of previous muon spin relaxation measurements in Dunsiger et al. ${ }^{47}$ and in Storchak et al. ${ }^{48}$ ).

The spacing $\Delta H_{1}$ shown in Fig. 6e scales as $L^{-2}$ and the values of $D$ extracted from the $n$ dependence of the resonant field spacings in the $L=48 \mathrm{~nm}$ epilayer (Fig. 6d) and from the $L$ dependence of $\Delta H_{1}$ (Fig. 6e) give the same $D=2.43 \pm 0.15 \mathrm{meV} \mathrm{nm}^{2}$. Identical value of the spin stiffness was also obtained from measurements in an optimized epilayer grown with the same doping and thickness of $18 \mathrm{~nm}$ in which we detected the frequencies $f_{0}$ and $f_{1}$ and applied equation (2). These measurements confirm the reliability of extracted values of the spin stiffness. We note that the SWR frequencies are determined with high accuracy in our measurements and that the indicated error bars in Fig. $4 \mathrm{~b}$ reflect the uncertainty of the film thickness (Supplementary Note 5). As shown in Fig. 4b, we observe a consistent, weakly increasing trend in $D$ with increasing doping and values of $D$ between 2 and $3 \mathrm{meVnm}^{2}$ in the studied ferromagnetic samples with nominal doping 3.8-9\%. (Note that apart from the difficulty of exciting magnetization precession in the very low and high-doped samples with stable easy axes, the measurements of $D$ were unfeasible on the lower doping side of the series because of the increasing damping and the corresponding inability to detect the higher SWR modes.) As mentioned in the discussion of Fig. 1 we have tested the inapplicability of the SWR experiments for the direct determination of the spin stiffness in thick non-uniform materials (Supplementary Fig. S5 and Supplementary Note 10). The observed number of modes and the field-dependent frequencies are non-systematic in these unoptimized materials and $\Delta H_{n}$ do not show the quadratic scaling with $n$ of the Kittel modes of equation (2) (see Supplementary Fig. S6 and Supplementary Note 10 for more details).

Similar to the Gilbert damping constant, our measured spin stiffness constant in the optimized $(\mathrm{Ga}, \mathrm{Mn})$ As epilayers is comparable to the spin stiffness in conventional transition metal ferromagnets ${ }^{49}$. The large values of the spin stiffness of the order $\mathrm{meV} \mathrm{nm}^{2}$ are also consistent with model Hamiltonian and $a b$ initio calculations ${ }^{28,50-52}$, which all consider or obtain the band structure of the ferromagnetic (Ga,Mn)As with merged valence and impurity bands ${ }^{6}$. On the other hand, for carriers localized in a narrow impurity band the expected spin stiffness would be small in a dilute moment system like (Ga,Mn)As, in which the magnetic coupling between remote Mn moments is mediated by the carriers ${ }^{53}$.

\section{Discussion}

We have presented a systematic study demonstrating the crucial role of the careful synthesis optimization for understanding the intrinsic semiconducting and magnetic properties of $(\mathrm{Ga}, \mathrm{Mn})$ As. Micromagnetic parameters of $(\mathrm{Ga}, \mathrm{Mn}) \mathrm{As}$ measured on our carefully optimized materials show values that are characteristic 
of common band ferromagnets and all the observed semiconducting and magnetic properties are consistent with the microscopically established electronic structure of $(\mathrm{Ga}, \mathrm{Mn}) \mathrm{As}$ with merged impurity and valence bands. By recognizing that the bands are merged, that is, overlapped and mixed, in the optimized ferromagnetic ( $\mathrm{Ga}, \mathrm{Mn})$ As materials, the distinction between 'valence' and 'impurity' bands becomes mere semantics with no fundamental physics relevance. Simultaneously, it is important to keep in mind that the moderate acceptor binding energy of $\mathrm{Mn}_{\mathrm{Ga}}$ shifts the insulator-to-metal transition to orders of magnitude higher doping densities than in the case of common shallow nonmagnetic acceptors. Disorder and correlation effects, therefore, have a comparatively more significant role in $(\mathrm{Ga}, \mathrm{Mn}) \mathrm{As}$ than in degenerate semiconductors with common shallow dopants and any simplified one-particle band picture of ferromagnetic $(\mathrm{Ga}, \mathrm{Mn})$ As can only represent a proxy to the electronic structure of the material.

\section{Methods}

Optimized materials synthesis. Because of the highly non-equilibrium nature of the heavily-doped ferromagnetic $(\mathrm{Ga}, \mathrm{Mn})$ As, the growth and post-growth annealing procedures have to be individually optimized for each $\mathrm{Mn}$-doping level to obtain films that are as close as possible to idealized uniform (Ga,Mn)As mixed crystals with the minimal density of compensating and other unintentional defects. An extensive set of characterization measurements has to accompany the synthesis to guarantee that the materials show systematic doping trends; monitoring the thermodynamic Curie point singularities is essential for assuring the uniformity and high magnetic quality of the materials ${ }^{11,15,54,55}$. When omitting the above procedures, extrinsic impurities and sample inhomogeneities can yield nonsystematic doping trends and conceal the intrinsic magnetic properties of (Ga,Mn)As.

Our $(\mathrm{Ga}, \mathrm{Mn})$ As layers were grown at the growth rate of $\sim 0.2$ monolayers per second. The Mn flux, and hence the nominal Mn doping $x$, was determined by measuring the ratio of the beam equivalent pressures (BEP) of $\mathrm{Mn}$ and Ga sources before each growth. The Mn content was cross-checked by secondary ion mass spectroscopy (SIMS) and by comparing the growth rates of GaAs and (Ga,Mn)As measured by the oscillations of the reflection high-energy electron diffraction. There are two critical growth parameters of (Ga,Mn)As: the substrate temperature, and the As-to- $(\mathrm{Ga}+\mathrm{Mn})$ flux ratio ${ }^{4,14,15,56}$. At the typical temperatures of $\sim 200^{\circ} \mathrm{C}$, neither an optical pyrometer nor a radiatively coupled temperature sensor are applicable. Instead, we use the GaAs band-edge spectrometer to measure the substrate temperature and the predictive substrate heater control to stabilize the temperature during the growth. For a given $\mathrm{As}:(\mathrm{Ga}+\mathrm{Mn})$ ratio, the substrate temperature fully determines the growth regime: the growth proceeds two-dimensionally at low temperatures, and turns irreversibly into the $3 \mathrm{D}$ growth mode when a critical temperature is exceeded. The scatter of the critical substrate temperature for given doping $x$ and $\mathrm{As}:(\mathrm{Ga}+\mathrm{Mn})$ ratio is remarkably small, typically less than $2^{\circ} \mathrm{C}$. For a larger As flux, the 2D/3D transition occurs at a higher temperature. The highest quality samples are grown in a narrow window around the 1:1 stoichiometric As: $(\mathrm{Ga}+\mathrm{Mn})$ ratio and at the substrate temperature approaching as close as possible from below the $2 \mathrm{D} / 3 \mathrm{D}$ critical temperature for given $x$. The As: $(\mathrm{Ga}+\mathrm{Mn})$ ratio was adjusted by the As-cell valve, and calibrated using the As-controlled reflection high-energy electron diffraction oscillations.

The next important factor determining the quality of the resulting (Ga,Mn)As materials are post-growth annealing conditions $4,15,54$. The common trends in annealing (at temperatures close to the growth temperature) suggest the presence of competing mechanisms. One mechanism yields the increase of $T_{\mathrm{C}}$ and is ascribed in a number of reports to the removal of charge and moment compensating interstitial Mn impurities ${ }^{57,58}$. The removal is slowed down by the growth of an oxide surface layer during annealing ${ }^{54}$ and an additional mechanism can eventually yield reduction of $T_{\mathrm{C}}$ after sufficiently long annealing times, depending on the annealing temperature. The origin of this detrimental mechanism may be in Mn clustering or in the competition between the nonequilibrium (Ga,Mn)As phase and the equilibrium MnAs second phase. Because of the competing mechanisms, the absolutely highest Curie temperature for the given nominal doping is achieved at intermediate annealing temperature and time. The remaining critical parameter of the synthesis is the epilayer thickness ${ }^{54}$. For a given nominal doping, the highest attainable $T_{\mathrm{C}}$ is reached only in thin films, typically thinner than $\sim 50 \mathrm{~nm}$.

An increase of $T_{\mathrm{C}}$ is not the only parameter followed to ascertain that a sample is of high quality. A key characterization tool is the thermodynamic Curie point singularity ${ }^{11}$. The development of sharply vanishing magnetization $M(T)$ at $T_{\mathrm{C}}$ and the onset of the singularity in $\mathrm{d} \rho / \mathrm{d} T$ are well correlated with increasing $T_{\mathrm{C}}$ and conductivity within the annealing sequence.
MO experiments. A Ti:sapphire laser was used as a light source. Laser pulses, with the time width of $200 \mathrm{fs}$ and the repetition rate of $82 \mathrm{MHz}$, were tuned to $1.64 \mathrm{eV}$ that is, above the semiconductor band gap, to excite magnetization dynamics by photon absorption. A schematic diagram of the experimental set-up is shown in Supplementary Fig. S7. The output of a femtosecond laser is divided into a strong pump pulse and a weak probe pulse that are focused to a same spot on the sample. The pump pulses were usually circularly polarized (with a helicity controlled by a wave plate) and the probe pulses were linearly polarized. The measured MO signals correspond to the probe-polarization rotation induced by the pump pulses. The experiment was performed close to the normal incidence geometry (angle of incidence $\theta_{\mathrm{i}}=2^{\circ}$ and $8^{\circ}$ for pump-and-probe pulses, respectively) with a sample mounted in a cryostat, which was placed between the poles of an electromagnet. All the MO data were measured at temperature of $15 \mathrm{~K}$, at pump excitation intensity $30-40 \mu \mathrm{J} \mathrm{cm}^{-2}$, and they correspond to the helicity-independent part of the measured signal ${ }^{40}$. The external magnetic field $H_{\text {ext }}$ was applied in the sample plane at an angle $\varphi_{\mathrm{H}}$ with respect to the [100] crystallographic direction in the sample plane (Supplementary Fig. S7). Before all time-resolved experiments, we always prepared the magnetization in a well-defined state by first applying a strong saturating magnetic field at an angle $\varphi_{\mathrm{H}}$ and then reducing it to the desired magnitude of $H_{\text {ext }}$. There are several microscopic mechanisms that can lead to a precession of magnetization due to the impact of pump laser pulse. In particular, very recently we reported on the precession of magnetization due to optical spintransfer torque ${ }^{40}$ and optical spin-orbit torque ${ }^{59}$. However, the most common mechanism, which is responsible for the oscillatory MO signals measured in the majority of $(\mathrm{Ga}, \mathrm{Mn})$ As samples at low excitation intensities, is the change of the sample magnetic anisotropy due to the pump-induced temperature increase $\mathrm{e}^{59}$ that is schematically shown in Supplementary Fig. S8. The most important point from the perspective of the present paper is that the precession of magnetization induced by the laser pulses is determined by the magnetic anisotropy of the sample, which makes this method an all-optical analogue to $\mathrm{FMR}^{60}$ (Supplementary Equations S2-S19).

Determination of micromagnetic parameters. All micromagnetic parameters reported here were deduced from a single MO pump-and-probe experiment. In particular, the anisotropy fields were determined by a fitting of the theoretically derived dependence of the precession frequency on the external magnetic field (Supplementary Equations S13-S15) to the experimentally obtained data (Supplementary Fig. S9). For all the investigated samples, we supplemented this method by two additional experimental techniques that provide information about the samples magnetic anisotropy, namely the probe-polarization dependence of the MO precession signal amplitude (Supplementary Fig. S10) and superconducting quantum interference device magnetometry (Supplementary Fig. S11). This enabled us to increase the precision of the magnetic anisotropy determination (Supplementary Fig. S12) even further. The Gilbert damping constant can be deduced from the precession signal damping using the LLG equation (Supplementary Equations S2-S7, S11 and S16) and the experimentally measured values of MO coefficients of the polar Kerr effect and magnetic liner dichroism (Supplementary Fig. S13); examples of the data are shown in Supplementary Fig. S14. Finally, the spin stiffness is obtained from the mutual spacing of the SWRs (Supplementary Fig. S15), which are present in the measured oscillatory MO signals (Supplementary Fig. S16). The inapplicability of this spin stiffness determination to thick (Ga,Mn)As epilayers (Supplementary Fig. S5) is illustrated in Supplementary Fig. S6.

\section{References}

1. Ohno, H. et al. (Ga,Mn)As: a new diluted magnetic semiconductor based on GaAs. Appl. Phys. Lett. 69, 363-365 (1996).

2. Hayashi, T., Tanaka, M., Seto, K., Nishinaga, T. \& Ando, K. III-V based magnetic(GaMnAs)/nonmagnetic(AlAs) semiconductor superlattices. Appl. Phys. Lett. 71, 1825-1827 (1997).

3. Van Esch, A. et al. Interplay between the magnetic and transport properties in the III-V diluted magnetic semiconductor $\mathrm{Ga}_{1-} \mathrm{Mn}_{x}$ As. Phys. Rev. B 56, 13103-13112 (1997).

4. Ohno, H. Making nonmagnetic semiconductors magnetic. Science 281, 951-956 (1998).

5. Jungwirth, T., Sinova, J., Mašek, J., Kučera, J. \& MacDonald, A. H. Theory of ferromagnetic (III,Mn)V semiconductors. Rev. Mod. Phys. 78, 809-864 (2006).

6. Mašek, J. et al. Microscopic analysis of the valence band and impurity band theories of (Ga,Mn)As. Phys. Rev. Lett. 105, 227202 (2010).

7. Burch, K. S. et al. Impurity band conduction in a high temperature ferromagnetic semiconductor. Phys. Rev. Lett. 97, 087208 (2006).

8. Tang, J.-M. \& Flatté, M. E. Magnetic circular dichroism from the impurity band in III-V diluted magnetic semiconductors. Phys. Rev. Lett. 101, 157203 (2008).

9. Dobrowolska, M. et al. Controlling the curie temperature in (Ga,Mn)As through location of the fermi level within the impurity band. Nat. Mater. 11, 444-449 (2012).

10. Samarth, N. Battle of the bands. Nat. Mater. 11, 360-361 (2012).

11. Novák, V. et al. Curie point singularity in the temperature derivative of resistivity in (Ga,Mn)As. Phys. Rev. Lett. 101, 077201 (2008). 
12. Walowski, J. et al. Intrinsic and non-local Gilbert damping in polycrystalline nickel studied by Ti: sapphire laser fs spectroscopy. J. Phys. D Appl. Phys. 41, 164016 (2008)

13. Liu, Y. et al. Optically induced magnetization dynamics and variation of damping parameter in epitaxial Co2MnSi Heusler alloy films. Phys. Rev. B 81, 094402 (2010).

14. Myers, R. C. et al. Antisite effect on hole-mediated ferromagnetism in (Ga,Mn)As. Phys. Rev. B 74, 155203 (2006).

15. Wang, M. et al. Achieving high Curie temperature in (Ga,Mn)As. Appl. Phys. Lett. 93, 132103 (2008).

16. Singley, E. J. et al. Electronic structure and carrier dynamics of the ferromagnetic semiconductor $\mathrm{Ga}_{1-x} \mathrm{Mn}_{x}$ As. Phys. Rev. 68, 165204 (2003).

17. Mack, S., Myers, R. C., Heron, J. T., Gossard, A. C. \& Awschalom, D. D. Stoichiometric growth of high Curie temperature heavily alloyed GaMnAs. Appl. Phys. Lett. 92, 192502 (2008).

18. Jungwirth, T. et al. Low-temperature magnetization of (Ga,Mn)As semiconductors. Phys. Rev. B 73, 165205 (2005).

19. Sinova, J. et al. Magnetization relaxation in (Ga,Mn)As ferromagnetic semiconductors. Phys. Rev. B 69, 085209 (2004).

20. Khazen, K. et al. Anisotropic magnetization relaxation in ferromagnetic $\mathrm{Ga}_{1-x} \mathrm{Mn}_{x} \mathrm{As}$ thin films. Phys. Rev. B 78, 195210(1)-195210(8) (2008).

21. Rappoport, T. G. et al. Anomalous behavior of spin wave resonances in

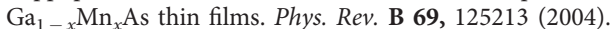

22. Zhou, Y.-Y. et al. Magnetic anisotropy, spin pinning, and exchange constants of (Ga,Mn)As films. IEEE Trans. Magn. 43, 3019-3021 (2007).

23. Liu, X., Zhou, Y. Y. \& Furdyna, J. K. Angular dependence of spin-wave resonances and surface spin pinning in ferromagnetic (Ga,Mn) As films. Phys. Rev. B 75, 195220 (2007).

24. Bihler, C., Schoch, W., Limmer, W., Goennenwein, S. T. B. \& Brandt, M. S. Spin-wave resonances and surface spin pinning in $\mathrm{Ga}_{1-x} \mathrm{Mn}_{x} \mathrm{As}$ thin films. Phys. Rev. B 79, 045205 (2009).

25. Potashnik, S. J. et al. Saturated ferromagnetism and magnetization deficit in optimally annealed (Ga,Mn)As epilayers. Phys. Rev. B 66, 012408 (2002).

26. Gourdon, C. et al. Determination of the micromagnetic parameters in (Ga,Mn)As using domain theory. Phys. Rev. B 76, 241301 (2007).

27. Wang, D. M. et al. Light-induced magnetic precession in (Ga,Mn)As slabs: Hybrid standing-wave Damon-Eshbach modes. Phys. Rev. B 75, 233308 (2007).

28. Werpachowska, A. \& Dietl, T. Theory of spin waves in ferromagnetic (Ga,Mn)As. Phys. Rev. B 82, 085204 (2010).

29. Zemen, J., Kučera, J., Olejnk, K. \& Jungwirth, T. Magneto crystalline anisotropies in $(\mathrm{Ga}, \mathrm{Mn}) \mathrm{As}$ : a systematic theoretical study and comparison with experiment. Phys. Rev. B 80, 155203 (2009).

30. Oiwa, A., Takechi, H. \& Munekata, H. Photoinduced magnetization rotation and precessional motion of magnetization in ferromagnetic ( $\mathrm{Ga}, \mathrm{Mn}) \mathrm{As}$. J. Supercond. Nov. Magn. 18, 9-13 (2005).

31. Wang, D. M. et al. Light-induced magnetic precession in (Ga,Mn)As slabs: Hybrid standing-wave damon-eshbach modes. Phys. Rev. B 75, 233308 (2007).

32. Takechi, H., Oiwa, A., Nomura, K., Kondo, T. \& Munekata, H. Light-induced precession of ferromagnetically coupled $\mathrm{Mn}$ spins in ferromagnetic (Ga,Mn)As. Phys. Status Solidi. C 3, 4267-4270 (2007).

33. Qi, J. et al. Coherent magnetization precession in GaMnAs induced by ultrafast optical excitation. Appl. Phys. Lett. 91, 112506 (2007).

34. Qi, J. et al. Ultrafast laser-induced coherent spin dynamics in ferromagnetic $\mathrm{Ga}_{1-x} \mathrm{Mn}_{x} \mathrm{As} / \mathrm{GaAs}$ structures. Phys. Rev. B 79, 085304 (2009).

35. Rozkotová, E. et al. Light-induced magnetization precession in GaMnAs. Appl. Phys. Lett. 92, 122507 (2008).

36. Rozkotová, E. et al. Coherent control of magnetization precession in ferromagnetic semiconductor (Ga,Mn)As. Appl. Phys. Lett. 93, 232505 (2008).

37. Hashimoto, Y. \& Munekata, H. Coherent manipulation of magnetization precession in ferromagnetic semiconductor $(\mathrm{Ga}, \mathrm{Mn})$ As with successive optical pumping. Appl. Phys. Lett. 93, 202506 (2008).

38. Hashimoto, Y., Kobayashi, S. \& Munekata, H. Photoinduced precession of magnetization in ferromagnetic (Ga,Mn)As. Phys. Rev. Lett. 100, 067202 (2008).

39. Kobayashi, S., Suda, K., Aoyama, J., Nakahara, D. \& Munekata, H. Photoinduced precession of magnetization in metal/(Ga,Mn)As systems. IEEE Trans. Magn. 46, 2470-2473 (2010).

40. Němec, P. et al. Experimental observation of the optical spin transfer torque. Nat. Phys. 8, 411-415 (2012).

41. Tesařová, N. et al. Direct measurement of the three dimensional magnetization vector trajectory in GaMnAs by a magneto-optical pump-and-probe method. Appl. Phys. Lett. 100, 102403 (2012).
42. Ingvarsson, S. et al. Role of electron scattering in the magnetization relaxation of thin $\mathrm{Ni}_{81} \mathrm{Fe}_{19}$ films. Phys. Rev. B 66, 214416 (2002).

43. Rantschler, J. O. et al. Effect of $3 d, 4 d$, and $5 d$ transition metal doping on damping in permalloy thin films. J. Appl. Phys. 101, 033911 (2007).

44. Gilmore, K., Idzerda, Y. U. \& Stiles, M. D. Spin-orbit precession damping in transition metal ferromagnets. J. Appl. Phys. 103, $07 \mathrm{D} 303$ (2008).

45. Kittel, C. Excitation of spin waves in a ferromagnet by a uniform rf field. Phys Rev. 110, 1295-1297 (1958).

46. Son, H. et al. Vertical gradient of magnetic anisotropy in the ferromagnetic semiconductor (Ga,Mn)As film. Appl. Phys. Lett. 96, 092105 (2010).

47. Dunsiger, S. R. et al. Spatially homogeneous ferromagnetism of (Ga, Mn)As. Nat. Mater. 9, 299-303 (2010).

48. Storchak, V. G. et al. Spatially resolved inhomogeneous ferromagnetism in GaMnAs diluted magnetic semiconductors: a microscopic study by muon spin relaxation. Phys. Rev. Lett. 101, 027202 (2008).

49. Collins, M. F., Minkiewicz, V. J., Natans, R., Passell, L. \& Shirane, G. Critical and spin-wave scattering of neutrons from iron. Phys. Rev. 179, 417-430 (1969).

50. König, J., Jungwirth, T. \& MacDonald, A. H. Theory of magnetic properties and spin-wave dispersion for ferromagnetic (Ga,Mn)As. Phys. Rev. B 64, 184423 (2001).

51. Brey, L. \& Gómez-Santos, G. Magnetic properties of GaMnAs from an effective Heisenberg Hamiltonian. Phys. Rev. B 68, 115206 (2003).

52. Bouzerar, G. Magnetic spin excitations in diluted ferromagnetic systems: the case of $\mathrm{Ga}_{1-x} \mathrm{Mn}_{x}$ As. Europhys. Lett. 79, 57007 (2007).

53. MacDonald, A. H., Schiffer, P. \& Samarth, N. Ferromagnetic semiconductors: moving beyond (Ga,Mn)As. Nat. Mater. 4, 195-202 (2005).

54. Olejník, K. et al. Enhanced annealing, high Curie temperature and low-voltage gating in (Ga,Mn)As: a surface oxide control study. Phys. Rev. B 78, 054403 (2008).

55. Jungwirth, T. et al. Systematic study of Mn-doping trends in optical properties of (Ga,Mn)As. Phys. Rev. Lett. 105, 227201 (2010).

56. Foxon, C. T. et al. The growth of high quality GaMnAs films by MBE. J. Mater Sci. 15, 727-731 (2004).

57. Yu, K. M. et al. Effect of the location of $\mathrm{Mn}$ sites in ferromagnetic $\mathrm{Ga}_{1-x} \mathrm{Mn}_{x} \mathrm{As}$ on its curie temperature. Phys. Rev. B 65, 201303 (2002).

58. Edmonds, K. W. et al. High Curie temperature GaMnAs obtained by resistance-monitored annealing. Appl. Phys. Lett. 81, 4991 (2002)

59. Tesarová, N. et al. Experimental observation of the optical spin-orbit torque (2012) Preprint at: http://arXiv.org/abs/1207.0307.

60. van Kampen, M. et al. All-optical probe of coherent spin waves. Phys. Rev. Lett. 88, 227201 (2002)

\section{Acknowledgements}

We acknowledge theoretical assistance of Pavel Motloch and support from EU ERC Advanced Grant No. 268066 and FP7-215368 SemiSpinNet, the Ministry of Education of the Czech Republic Grants No. LM2011026, the Grant Agency of the Czech Republic Grant No. 202/09/H041 and P204/12/0853, the Charles University in Prague Grant No. SVV-2012-265306 and 443011, the Academy of Sciences of the Czech Republic Preamium Academiae and US grants ONR-N000141110780, NSF-MRSEC DMR0820414, NSF-DMR-1105512.

\section{Author contributions}

Sample preparation: V.N., K.O., R.P.C.; experiments and data analysis: N.T., P.N., E.R D.B., P. M., H.R., V.N., K.O, T.J., B.L.G.; data modelling: P.N., F.T., H.R., K.O., V.N.; theory: T.J., J.S.; writing: T.J., P.N., J.S.; project planning: P.N., V.N., T.J.

\section{Additional information}

Supplementary Information accompanies this paper at http://www.nature.com/ naturecommunications

Competing financial interests: The authors declare no competing financial interests

Reprints and permission information is available online at http://npg.nature.com/ reprintsandpermissions/

How to cite this article: Němec, P. et al. The essential role of carefully optimized synthesis for elucidating intrinsic material properties of (Ga,Mn)As. Nat. Commun. 4:1422 doi: 10.1038/ncomms2426 (2012). 